\title{
Bilans d'éléments minéraux dans de petits bassins versants forestiers de Galice (NW Espagne)
}

\author{
Etienne Dambrine ${ }^{\mathrm{a}, *}$, José Antonio Vega ${ }^{\mathrm{c}}$, Teresa Taboada ${ }^{\mathrm{b}}$, Luis Rodriguez ${ }^{\mathrm{b}}$, \\ Cristina Fernandez ${ }^{\mathrm{c}}$, Felipe Macias ${ }^{\mathrm{b}}$ et José Maria Gras ${ }^{\mathrm{c}}$ \\ ${ }^{a}$ Équipe Cycles Biogéochimiques, INRA, 54280 Champenoux, France \\ ${ }^{\mathrm{b}}$ Departamento de Edafologia, Universidad de Biologia, 15706 Santiago, Espagne \\ ${ }^{\mathrm{c}}$ Centro de Investigacion Forestal, Lourizan, 36080 Pontevedra, Espagne
}

(Reçu le 15 février 1999 ; accepté le 3 mai 1999)

\begin{abstract}
Résumé - Afin d'estimer la pérennité de l'alimentation minérale de plantations forestières de Pinus pinaster et Eucalyptus globulus de forte productivité en Galice, nous avons calculé des bilans (entrées-sorties) d'éléments minéraux dans trois petits bassins versants. Dans chacun de ces trois bassins, les sorties par drainage de $\mathrm{Ca}, \mathrm{Mg}$ et $\mathrm{K}$ sont inférieures aux entrées par les pluies. Cette particularité est attribuée à la relative pauvreté en cations alcalino-terreux des roches mères, au très faible drainage de sulfate et nitrate lié à l'absence de pollution atmosphérique, et surtout à la forte immobilisation forestière qui tire directement profit des apports par les pluies. L'exploitation forestière actuelle des peuplements de pins et d'eucalyptus conduit à des bilans fortement négatifs de $\mathrm{Ca}, \mathrm{Mg}$ et $\mathrm{K}$, mais qui diffèrent peu entre ces peuplements. La différence majeure provient du mode d'exploitation, selon que les arbres sont ou non écorcés en forêt, et que la litière est exportée. La libération par altération minérale d'un flux relativement important de Ca et $\mathrm{K}$ est nécessaire pour compenser ces pertes. Les estimations du flux d'altération conduisent à penser que ce flux est suffisant dans le bassin versant sur granodiorite. Dans les bassins versants sur granite, ce flux est vraisemblablement insuffisant en ce qui concerne le Ca, mais des mesures complémentaires sont nécessaires pour pouvoir l'affirmer. Les bilans d'azote montrent qu'une forte fixation symbiotique est indispensable pour assurer la pérennité de la production forestière.
\end{abstract}

bassin versant / Eucalyptus globulus / Pinus pinaster / altération / Galice

Abstract - Budgets of mineral elements in small forested catchments in Galicia (NW Spain). In order to assess the sustainability of mineral supply to fast growing Eucalyptus globulus and Pinus pinaster stands in Galice (NW Spain), we calculated (input-output) nutrient budgets in three small catchments: the Castrove and Jabali catchments with eucalyptus on a base poor granite and the Arcos catchment with pine on granodiorite. In each catchment, the annual drainage output was lower than the rainwater input for $\mathrm{Ca}$, $\mathrm{Mg}$ and $\mathrm{K}$. This particularity is attributed to (a) the relative low content of $\mathrm{Ca}$ and $\mathrm{Mg}$ of the parent material, (b) the very low concentrations of $\mathrm{NO}_{3}$ and $\mathrm{SO}_{4}$ of drainage water in an area with very low level of atmospheric pollution, and (c) to the strong mineral uptake of fast growing stands. The present management of forests leads to negative budgets, both in the eucalyptus and pine catchments. But the traditional management of pine stands including the harvest of needles and branches was much more impoverishing than the present management of eucalyptus stands, where timber is the only harvested product. Various estimates of the weathering rate suggest that this flux is sufficient to compensate for the losses in the catchment on granodiorite but probably insufficient on granite. Since nitrogen deposition is extremely low, and forest fires are quite frequent, the symbiotic fixation of large amounts of nitrogen is needed in order to ensure the sustainability of forest growth.

catchment / Eucalyptus globulus / Pinus pinaster / weathering / Galicia

* Correspondance et tirés à part

Tél. 0383394041 ; Fax. (33) 0383394069 ; e-mail : dambrine@ nancy.inra.fr 


\section{INTRODUCTION}

En Galice (NW Espagne), l'eucalyptus (Eucalyptus globulus) a été planté massivement après guerre dans toute la zone côtière pour alimenter les industries papetières (figure 1). Sa production exceptionnelle, son formidable potentiel de régénération et sa bonne résistance au feu font qu'il occupe actuellement environ 100000 ha soit en peuplement pur, soit en mélange avec le pin maritime. Les effets des plantations d'eucalyptus sur la fertilité chimique des sols ont été évalués par divers auteurs [5]. En Galice, la comparaison des taux de saturation de 500 échantillons de sols prélevés sous différentes végétations forestières ne montre pas de différences significatives [9]. Alvarez et al. [2] comparent cependant la composition de la solution du sol (entre 5 et $35 \mathrm{~cm}$ de profondeur) dans des peuplements d'eucalyptus, de pins et de chênes adjacents et observent des concentrations moyennes en $\mathrm{Ca}$ et $\mathrm{Mg}$ plus faibles sous les plantations d'eucalyptus. Les teneurs les plus élevées en $\mathrm{Al}$ et en carbone organique dissous s'observent par contre sous les peuplements de chêne. L'étude par Bara [4] d'une chronoséquence de peuplements montre un abaissement très net des teneurs en $\mathrm{Ca}, \mathrm{K}$ et $\mathrm{Mn}$ échangeables des sols et du $\mathrm{pH}$ lorsque croît l'âge du peuplement. Au Portugal, sous des plantations d'Eucalyptus globulus, une étude des stocks d'éléments minéraux [35] indique un appauvrissement des horizons minéraux du sol en $\mathrm{Ca}$ et en $\mathrm{Mg}$ échangeable au profit d'une accumulation dans la biomasse forestière et l'humus. Cette redistribution n'apparaît pas dans un peuplement de chêne voisin, dont le bois n'est pas exploité.

Sur le long terme, la réserve d'éléments échangeables des sols est alimentée par deux sources: les apports atmosphériques et l'altération des minéraux des sols. Elle se vide à travers les exportations dans la biomasse et le drainage profond. L'équilibre entre ces flux définit donc la pérennité de cette réserve. Sur un plus court terme, les pertes occasionnelles, provoquées par les incendies ou les coupes rases, mais aussi des flux internes à l'écosystème conduisant à la redistribution d'éléments minéraux d'un compartiment à l'autre (chutes de litière, translocations internes à l'arbre, minéralisation) doivent être pris en compte.

Pour évaluer le bilan de fertilité à long terme des plantations d'eucalytus, nous nous sommes placés dans un dispositif expérimental de bassins versants à végétation contrastée, équipés depuis 1987 pour mesurer des bilans hydrologiques par le Centre de Recherches Forestières de Lourizan [20].

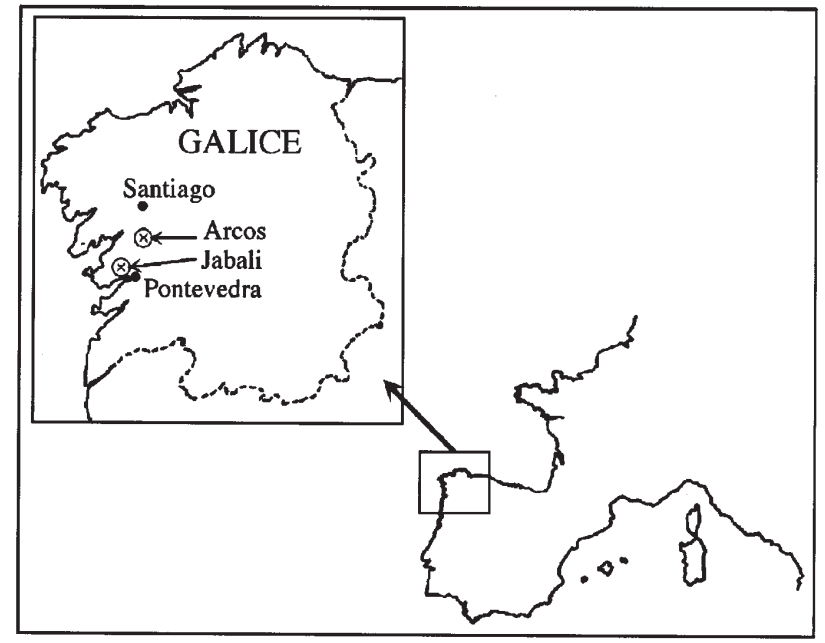

Figure 1. Situation de la région étudiée.

\section{MATÉRIEL ET MÉTHODES}

\subsection{Sites d'étude}

La pinède du bassin versant d'Arcos occupe une petite cuvette (6,7 ha) peu marquée, située dans le massif granodioritique de Puente Caldelas, sur la commune de Arcos de la Condesa, à $20 \mathrm{~km}$ au Nord de Pontevedra. Ce batholite de granodiorite est composé de $29,5 \%$ de quartz, 25,5\% de microcline, $30 \%$ de plagioclase (An $27 \%), 10,5 \%$ de biotite et $2 \%$ de muscovite. L'altitude moyenne du bassin versant est de $200 \mathrm{~m}$ et il est distant de l'océan d'environ $5 \mathrm{~km}$ à vol d'oiseau. La pluviométrie moyenne mesurée sur le site au cours des années 1993-1995 est de $1500 \mathrm{~mm}$. On y distingue une zone de crête peu étendue, des versants en pente faible $(<10 \%)$ représentant environ $35 \%$ de la surface et un bas fond le long du ruisseau. Ce bassin versant est couvert par une forêt de pin (Pinus pinaster) âgée d'une quarantaine d'années, très productive. Les peuplements de la zone de crête sont fortement éclaircis. Une coupe rase a eu lieu en 1991 sur environ $25 \%$ de la surface du bassin versant. Traditionnellement, ces forêts ont été très intensément exploitées, pour le combustible (les branches et les chutes de litière), le bois d'oeuvre et la litière animale (les fougères) [7]. Actuellement, troncs, branches et cônes sont récoltés mais le ramassage des litières a été abandonné.

La plantation d'eucalyptus occupe le bassin versant de Jabali, qui domine la Ria de Pondevedra, à moins de $1 \mathrm{~km}$ de la mer. Sa superficie est de 194 ha. Le sous-bassin versant de Castrove qui s'étage entre 348 et $445 \mathrm{~m}$ 
d'altitude, pour une superficie de 9,9 ha, en occupe la zone sommitale. La pluviométrie moyenne annuelle mesurée depuis 7 années au sommet du bassin versant approche $2000 \mathrm{~mm}$. La roche mère est un granite à 2 micas, pauvre en $\mathrm{Ca}$ et $\mathrm{Mg}$, d'age hercynien [6]. En moyenne il est composé de 30,8\% de quartz, 13,3\% de muscovite, $27.8 \%$ de microcline, $3.2 \%$ de biotite et 24,7 d'albite-oligoclase acide. La zone sommitale d'environ 3 ha de superficie est occupée par des sols peu épais et de larges surfaces de roches nues y affleurent. La pente du versant est accentuée $(30 \%)$. Les sols y sont plus épais, jusqu'à $1 \mathrm{~m}$ de profondeur dans le bassin versant supérieur de Castrove, jusqu'à plusieurs mètres de profondeur dans la partie basse du bassin de Jabali. Ces bassins étaient occupés en 1988 par un peuplement productif monospécifique d'eucalyptus (Eucalyptus globulus) âgé de 15 ans, planté sur une ancienne lande à ajonc. Durant l'année 1988-1989, deux incendies consécutifs ont en quasi-totalité détruit le sous-bois et les houppiers des arbres. Une proportion notable d'arbres moururent mais la majorité résistèrent et reprirent très rapidement leur croissance. Les eucalyptus du bassin versant de Castrove et une proportion mineure de ceux couvrant le bassin versant de Jabali furent récoltés en 1991-1992. De tels incendies précédant la coupe rase sont communs dans les peuplements d'eucalyptus en Galice. Lors de l'exploitation, les troncs ne sont pas écorcés en forêt.

\subsection{Sols}

Afin de caractériser la couverture pédologique du bassin versant d'Arcos, une carte des sols à l'échelle du $1 / 10000$ a été levé par sondages à la tarière. Puis trois profils types situés sur la crête, le bas de pente et le bas fond ont été décrits et échantillonnés. Les sols des bassins versants de Castrove et Jabali ont seulement été reconnus à partir de sondages ponctuels et des talus de pistes. Deux profils ont été décrits, échantillonnés et analysés, l'un sur la crête du bassin versant de Castrove, et l'autre sur le versant en pente forte du bassin de Jabali. Sur ces échantillons, nous avons mesuré la granulométrie, le $\mathrm{pH}$ eau, les $\mathrm{C}$ et $\mathrm{N}$ totaux, le $\mathrm{P}$ assimilable (méthode Dyer), la capacité d'échange au $\mathrm{pH}$ du sol et les cations échangeables par $\mathrm{BaCl}_{2}(0,1 \mathrm{~N})$, le fer et l'aluminium amorphes extractibles par le réactif de Tamm. La minéralogie des limons, des argiles $(<2 \mu \mathrm{m})$ et des argiles fines $(<0,2 \mu \mathrm{m})$ a été évaluée par diffraction des rayons $X$ après séparation et traitements usuels suivant les méthodes décrites par Robert [37]. La fraction sable + limon, isolée par tamisage puis sédimentation après épuisement des argiles, a été analysée après fusion alcaline.

\subsection{Biomasse}

Dans chacune des unités topographiques du bassin versant d'Arcos, un inventaire des hauteurs totales et des diamètres à $1,3 \mathrm{~m}$ et à la souche a été réalisé sur des surfaces variant entre 900 (crête) et $2000 \mathrm{~m}^{2}$ (pente et bas fond). Douze arbres, représentatifs de la population sur pied ont été abattus et nous avons mesuré le volume des troncs et poids de branches et d'aiguilles. Par régression multiple, nous avons calculé les relations entre volume de tronc, hauteur totale et surface terrière d'une part, et entre biomasse de branches et d'aiguilles et surface terrière d'autre part. La biomasse de tronc a été calculée en utilisant une valeur d'infradensité de 0,46 [29]. Les biomasses des peuplements sur pied ont été calculées en appliquant ces relations à l'inventaire des peuplements. Pour évaluer la production annuelle ainsi que la biomasse exportée au cours de la révolution, lors des éclaircies et de la coupe finale, nous avons utilisé les tables de production de Pinus pinaster pour la Galice [41].

Pour évaluer la production de branches récoltée au cours des éclaircies, nous avons considéré que le poids à l'hectare de branches sur pied est approximativement constant à partir de 20 ans et qu'en conséquence la proportion de branches récoltées à chaque éclaircie est égale au produit de la proportion d'arbres enlevés par la biomasse de branches actuelle. On obtient la valeur de 2,25 fois la biomasse de branche actuelle. En ce qui concerne les aiguilles, les chutes de litière annuelles représentent les exportations maximales. Une approximation raisonnable des quantités récoltables a été obtenue en considérant que la durée moyenne de vie d'une aiguille sur l'arbre est de 2 ans. La chute actuelle de litière peut alors être calculée comme la moitié de la biomasse d'aiguilles sur pied. Sur la révolution, nous avons fait l'hypothèse que les chutes annuelles de litière qui ont pu être traditionnellement récoltées sont égales aux chutes de litière actuelles, c'est-à-dire, à la moitié de la biomasse actuelle d'aiguilles. Les minéralomasses ont été calculées en multipliant les biomasses par des teneurs moyennes en $\mathrm{N}, \mathrm{P}$, $\mathrm{K}$, Ca et $\mathrm{Mg}$ mesurées sur 12 rondelles (bois et écorce) de tronc prélevées entre 2 et $4 \mathrm{~m}$ de hauteur et 3 échantillons moyens de branches grossières, branches fines et litières [11].

Dans les bassins versants de Castrove et Jabali, afin d'évaluer la biomasse sur pied avant les incendies, et la production de bois, nous avons utilisé les densités, hauteurs dominantes et surfaces terrières mesurées en 1987 $[20,21]$ ainsi que les tables de production d'eucalyptus pour la Galice côtière [17]. La minéralomasse des troncs a été évaluée en multipliant les biomasses par la concentrations moyenne de rondelles de bois et d'écorce 
prélevées à la base, à mi-hauteur et à la cime de deux arbres adultes.

\subsection{Précipitation et débit hydrologique et chimique à l'exutoire}

Les précipitations ont été enregistrées en continu par des pluviomètres à augets basculants dans des clairières des bassins versants de Castrove et Arcos, et collectées par des entonnoirs alimentant des bidons en polyéthylène placées à l'abri de la lumière, situés à proximité. Les mesures de température, d'hygrométrie et de vitesse du vent, enregistrées sur les mêmes sites, ont été utilisées pour calculer l'ETP. Les débits des cours d'eau ont été enregistrés en continu dans des seuils jaugeurs maçonnés. Les eaux ont été échantillonnées par un préleveur automatique asservi au débit [20]. Après filtration $(0,45 \mu \mathrm{m})$ et mesure du $\mathrm{pH}, \mathrm{Ca}$ et $\mathrm{Mg}$ ont été analysés par spectrophotométrie d'absorption atomique en présence de lanthane, et $\mathrm{K}$ et $\mathrm{Na}$ par émission. Ces analyses ont été réalisées fréquemment en 1987, épisodiquement entre 1987 et 1993 et systématiquement entre octobre 93 et juillet 95. Des analyses complètes comportant $\mathrm{pH}$, cations (par les méthodes susmentionnées), anions ( $\mathrm{SO}_{4}$, $\mathrm{NO}_{3}$ et $\mathrm{Cl}$ par chromatographie ionique) et silice (colorimétrie) ont été systématiquement effectuées à partir de 1994. Un bilan annuel complet pour tous les éléments a été effectué entre le 29/4/94 et le 26/4/95, correspondant à des périodes de sécheresse relative.

\section{RÉSULTATS}

\subsection{Caractères généraux des sols}

Trois types de sols s'individualisent sur le bassin versant d'Arcos, essentiellement en fonction de la topographie. Sur les crêtes (Arcos 1), on trouve des profils $A C$ ou $A(B) C$ peu épais, et dont l'horizon $\mathrm{A}$, riche en matière organique, peut atteindre $40 \mathrm{~cm}$ d'épaisseur. Il s'agit de rankers atlantiques (alocrisols humiques [1] ou umbric leptosols et humic regosols $[16,27])$. Un horizon $(B)$ cambique d'épaisseur variant entre $20 \mathrm{~cm}$ et $1 \mathrm{~m}$ se développe sur le versant (Arcos 2). Les sols du bas fond sont de type $A C$ ou $A(B) C$, l'horizon A cumulique, de couleur foncée pouvant atteindre $120 \mathrm{~cm}$ d'épaisseur (Arcos 3). La texture des horizons $A$ et $B$ est sableuse, Le taux d'argile varie entre 8 et $17 \%$ (tableau I). Le taux de carbone est élevé dans les horizons $A$. Le rapport $C / N$ est bas dans les sols du bas fond mais il s'élève sensiblement dans les horizons de surface des sols du versant et de la crête. Le pH des horizons augmente avec la profondeur, de 4,2 en $A_{1}$ à approximativement 5 en $C$, sauf dans le bas fond où il reste stable sur tout le profil, autour de 5,1 . Capacité d'échange et taux de saturation sont bas sauf dans l'horizon $C$ du sol de versant.

Les mêmes types de sols ont été observés dans les bassins versants de Castrove et Jabali (tableau I). Cependant, les pentes plus accentuées, ainsi que le caractère moins altérable du granite confèrent aux sols une charge en éléments grossiers beaucoup plus élevée. La teneur en sable dépasse systématiquement $70 \%$, celle en argile est inférieure à $10 \%$. Le pH varie entre 4,2 en surface et 4,7 en $C$. La capacité d'échange est basse, inférieure à $4 \mathrm{cmol}(+) \mathrm{kg}^{-1}$, les taux de saturation sont inférieurs à $7 \%$ et le $\mathrm{Ca}$ echangeable est inférieur ou égal à $0,01 \mathrm{cmol}(+) \mathrm{kg}^{-1}$ dans tous les horizons.

Les teneurs en $\mathrm{CaO}$ total de la fraction limon + sable sont $\leq 0,5 \%$ à Arcos et $\leq 0,3 \%$ à Jabali, ce qui confirme la nature essentiellement sodique des plagioclases. Cette différence se retrouve pour le $\mathrm{Mg}$, et reflète la nature magnésienne (Arcos) ou alumineuse (Jabali) des minéraux micacés. Les teneurs en $\mathrm{P}_{2} \mathrm{O}_{5}$ varient entre 0,1 et $0,3 \%$.

Dans les trois bassins versants, nous avons observé des racines dans l'arène profonde et jusque dans les fractures des roches peu altérées. Cet enracinement profond justifie l'utilisation de bassin versants pour des bilans de fertilité.

\subsection{Minéralogie des sols (tableau II)}

Les argiles fines des sols du bassin versant d'Arcos sont de la gibbsite et des édifices 1:1 de type kaolinite désordonnée. Les minéraux 1:1 dominent nettement dans les sols du versant et du bas fond. Dans l'horizon $C$ profond du sol Arcos 2, marqué par des bariolages de ségrégation du fer et voisin du niveau de la nappe apparaît un minéral gonflant de type smectite (non indiqué sur le tableau II). Ce minéral n'apparaît ni sur la crête, ni dans le bas fond. Seules des traces de vermiculite apparaissent dans la fraction argile fine. La proportion relative de gibbsite et minéraux 1:1 dans les argiles fines diffère peu de celle des argiles totales, mais les argiles totales sont fortement enrichies en vermiculite. La fermeture de ces minéraux après saturation $K$ est incomplète dans tous les niveaux de surface ( $A$ et $B$ ) ce qui témoigne de leur aluminisation. La fraction limon est riche en minéraux 1:1 et en vermiculite et en revanche pauvre en gibbsite.

À Jabali et Castrove, la gibbsite domine dans les fractions argile fine et argile totale, tandis que les minéraux 1:1 et les micas dominent dans la fraction limon. 


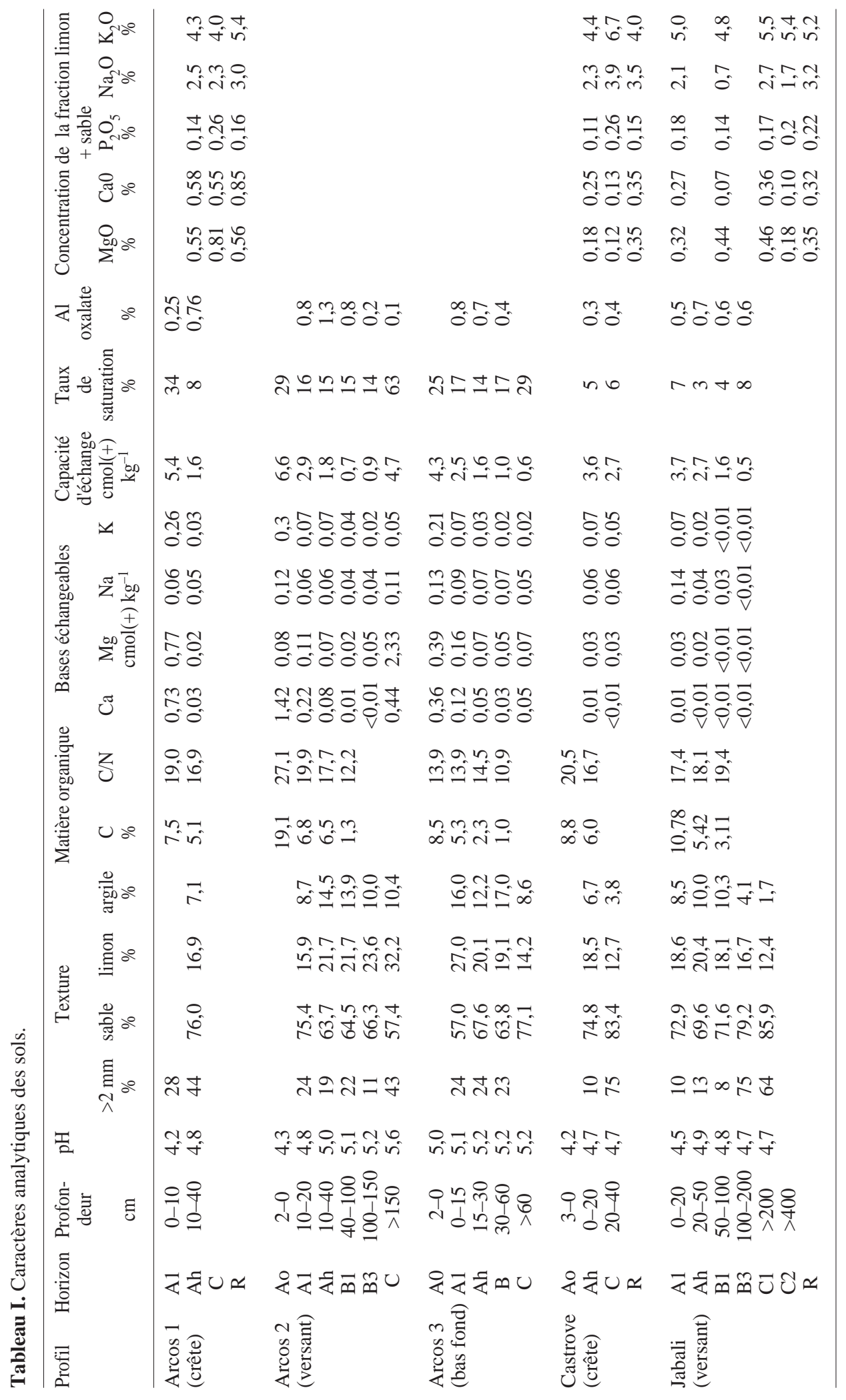




\subsection{Biomasse, minéralomasse et exportation d'éléments minéraux par les récoltes à Arcos}

Les caractères principaux des peuplements sont indiqués sur le tableau III.

Les peuplements de la zone basse et de la pente ont tous les deux une hauteur dominante proche de $25 \mathrm{~m}$ à 43 ans pour un volume sur pied de l'ordre de $440 \mathrm{~m}^{3}$ $\mathrm{ha}^{-1}$. Ces peuplements se situent dans une classe de production élevée de la table de production utilisée en Galice. Par comparaison, ces peuplements rentreraient dans la classe I de la table de Décourt [13] adaptée au Pin maritime des Landes. Dans la zone de crête, la densité est proche de celle de la zone basse, mais la hauteur dominante $(20 \mathrm{~m})$ et le volume sur pied $\left(309 \mathrm{~m}^{3} \mathrm{ha}^{-1}\right)$ sont plus faibles. Suivant les peuplements, le poids de branches à l'hectare varie entre 26 et 37 tonnes, tandis que le poids d'aiguilles varie entre 3,8 et 5,4 tonnes. En moyenne sur le bassin versant, et suivant l'intensité des éclaircies effectuées, la production cumulée de matière sèche exportable au cours de la révolution forestière représente entre 6 et $6,5 \mathrm{t} \mathrm{ha}^{-1} \mathrm{an}^{-1}$ de bois dont environ $0,4 \mathrm{t} \mathrm{ha}^{-1} \mathrm{an}^{-1}$ d'écorce, entre 1,5 et $2 \mathrm{t} \mathrm{ha}^{-1} \mathrm{an}^{-1} \mathrm{de}$ branches, et 2,5 à $3,5 \mathrm{t} \mathrm{ha}^{-1} \mathrm{an}^{-1}$ de litière, c'est-à-dire un total de 10 à $12 \mathrm{t} \mathrm{ha}^{-1} \mathrm{an}^{-1}$ de matière sèche.

Les troncs du peuplement du bas fond et du versant immobilisent annuellement environ $3 \mathrm{~kg}$ d'N, K et Ca, $0,3 \mathrm{~kg}$ de $\mathrm{P}$ et $1,5 \mathrm{~kg}$ de $\mathrm{Mg}$ par hectare pour une production moyenne annuelle de 4,7 tonnes de matière sèche. Suivant l'intensité des éclaircies, l'exportation moyenne

Tableau II. Minéralogie semi-quantitative des fractions argile fine $(<0,2 \mu \mathrm{m})$, argile totale $(<2 \mu \mathrm{m})$ et limon $(2-20 \mu \mathrm{m})$ des sols. (1:1 : kaolinite et halloisite ; $\mathrm{T}:$ traces, $\mathrm{X}:$ peu abondant, $\mathrm{XX}:$ abondant, $\mathrm{XXX}$ : très abondant).

\begin{tabular}{|c|c|c|c|c|c|c|c|c|c|c|c|c|c|}
\hline \multirow[t]{2}{*}{ Profil } & \multirow[t]{2}{*}{ Horizon } & \multirow{2}{*}{$\begin{array}{l}\text { Profon- } \\
\text { deur } \\
\mathrm{cm}\end{array}$} & \multicolumn{3}{|c|}{ Gibbsite } & \multicolumn{3}{|c|}{$1: 1$} & \multicolumn{3}{|c|}{ Vermiculite (+/-Al) } & \multicolumn{2}{|c|}{ Mica } \\
\hline & & & $<0,2 \mu \mathrm{m}$ & $<2 \mu \mathrm{m}$ & $2-20 \mu \mathrm{m}$ & $<0,2 \mu \mathrm{m}$ & $<2 \mu \mathrm{m}$ & $2-20 \mu \mathrm{m}$ & $<0,2 \mu \mathrm{m}$ & $<2 \mu \mathrm{m}$ & $2-20 \mu \mathrm{m}$ & $<2 \mu \mathrm{m}$ & $2-20 \mu \mathrm{m}$ \\
\hline Arcos 1 (crête) & $\mathrm{Ah}$ & $10-40$ & $X X$ & $\mathrm{XX}$ & $\mathrm{T}$ & $\mathrm{X}$ & $X X$ & $\mathrm{XX}$ & & $X X$ & $X X$ & $\mathrm{~T}$ & $\mathrm{X}$ \\
\hline \multirow[t]{4}{*}{ Arcos 2 (versant) } & A1 & $10-20$ & $\mathrm{X}$ & $\mathrm{X}$ & & $X X$ & $X X$ & $\mathrm{XX}$ & $\mathrm{T}$ & $\mathrm{XX}$ & $\mathrm{XX}$ & $\mathrm{T}$ & $\mathrm{X}$ \\
\hline & $\begin{array}{l}\mathrm{Ah} \\
\mathrm{B} 1\end{array}$ & $\begin{array}{c}10-40 \\
40-100\end{array}$ & XX & $\mathrm{XX}$ & & XX & $\mathrm{XX}$ & $\mathrm{XX}$ & $\mathrm{T}$ & $\mathrm{XX}$ & $\mathrm{XX}$ & - & $\mathrm{X}$ \\
\hline & B3 & $100-150$ & $X X$ & $\mathrm{X}$ & $\mathrm{T}$ & XXX & $\mathrm{XX}$ & $\mathrm{XX}$ & $\mathrm{T}$ & $\mathrm{X}$ & $\mathrm{XX}$ & $\mathrm{T}$ & $\mathrm{X}$ \\
\hline & $\mathrm{C}$ & $>150$ & - & - & $\mathrm{T}$ & $\mathrm{XX}$ & $\mathrm{XXX}$ & $\mathrm{XXX}$ & - & - & - & $X$ & $\mathrm{X}$ \\
\hline \multirow{4}{*}{$\operatorname{Arcos} 3$ (bas fond) } & $\mathrm{A} 1$ & $0-15$ & $\mathrm{~T}$ & $\mathrm{~T}$ & & $\mathrm{XX}$ & $X X$ & $X$ & $\mathrm{~T}$ & $\mathrm{XX}$ & $\mathrm{X}$ & - & $X$ \\
\hline & $\mathrm{Ah}$ & $15-30$ & $\mathrm{X}$ & $\mathrm{T}$ & & $X X$ & $X X$ & $X X$ & $\mathrm{~T}$ & $X X$ & XXX & - & $\mathrm{X}$ \\
\hline & B & $30-60$ & $\mathrm{X}$ & $X$ & & XX & XX & XX & $\mathrm{T}$ & XX & XX & - & $\mathrm{X}$ \\
\hline & $\mathrm{C}$ & $>60$ & $\mathrm{XX}$ & $\mathrm{XX}$ & $\mathrm{T}$ & XXX & $\mathrm{XX}$ & $\mathrm{XX}$ & $\mathrm{T}$ & $X$ & $\mathrm{XX}$ & $\mathrm{T}$ & $\mathrm{X}$ \\
\hline Castrove (crête) & $\mathrm{C}$ & $20-40$ & $\mathrm{XXX}$ & $\mathrm{XXX}$ & $\mathrm{T}$ & XX & XX & XX & - & $\mathrm{X}$ & $\mathrm{T}$ & $\mathrm{X}$ & $\mathrm{X}$ \\
\hline \multirow[t]{4}{*}{ Jabali (versant) } & $\mathrm{Ah}$ & $20-50$ & $\mathrm{XXX}$ & $\mathrm{XX}$ & $\mathrm{T}$ & $\mathrm{X}$ & $X X$ & $\mathrm{XX}$ & $\mathrm{T}$ & $\mathrm{X}$ & $\mathrm{T}$ & $\mathrm{T}$ & $\mathrm{XX}$ \\
\hline & B1 & $50-100$ & XXX & XXX & $X$ & $X$ & XX & XXX & - & $\mathrm{T}$ & $\mathrm{T}$ & $\mathrm{T}$ & XX \\
\hline & B3 & $100-200$ & XXX & XXX & $X$ & $\mathrm{~T}$ & $\mathrm{~T}$ & $X X$ & - & - & $\mathrm{T}$ & $\mathrm{T}$ & $X$ \\
\hline & $\mathrm{C} 1$ & $>200$ & $\mathrm{XXX}$ & XXX & $\mathrm{X}$ & $\mathrm{T}$ & $\mathrm{T}$ & $X X$ & - & - & $\mathrm{T}$ & - & $\mathrm{X}$ \\
\hline
\end{tabular}

Tableau III. Caractères dendrométriques et biomasse des peuplements d'eucalyptus et de pin des bassins versants de Castrove et Arcos.

\begin{tabular}{|c|c|c|c|c|c|c|c|c|c|}
\hline Site/espèce & Parcelle & Densité & âge & $\begin{array}{c}\text { Hauteur } \\
\text { dominante } \\
\text { m }\end{array}$ & $\begin{array}{l}\text { Surface } \\
\text { terrière } \\
\mathrm{m}^{2} \mathrm{ha}^{-1}\end{array}$ & $\begin{array}{l}\text { bois } \\
\mathrm{t} \mathrm{ha}^{-1}\end{array}$ & $\begin{array}{l}\text { écorce } \\
\mathrm{t} \mathrm{ha}^{-1}\end{array}$ & $\begin{array}{c}\text { Biomasse } \\
\text { aiguilles } \\
\mathrm{t} \mathrm{ha}^{-1}\end{array}$ & $\begin{array}{l}\text { branches } \\
\text { t ha }^{-1}\end{array}$ \\
\hline Castrove/Eucalytus & $\begin{array}{c}\text { Bas fond et versant } \\
\text { Crête }\end{array}$ & $\begin{array}{c}1400 \\
200\end{array}$ & $\begin{array}{l}15 \\
15\end{array}$ & $\begin{array}{l}28 \\
15\end{array}$ & $\begin{array}{c}23 \\
5\end{array}$ & $\begin{array}{c}146 \\
-\end{array}$ & $\begin{array}{c}19 \\
-\end{array}$ & & \\
\hline Arcos/Pin & $\begin{array}{c}\text { Bas fond et versant } \\
\text { Crête }\end{array}$ & $\begin{array}{l}315 \\
275\end{array}$ & $\begin{array}{l}43 \\
43\end{array}$ & $\begin{array}{l}26 \\
20\end{array}$ & $\begin{array}{l}48 \\
40\end{array}$ & $\begin{array}{l}184 \\
142\end{array}$ & $\begin{array}{l}12,9 \\
10,0\end{array}$ & $\begin{array}{l}4,6 \\
3,9\end{array}$ & $\begin{array}{l}31 \\
26\end{array}$ \\
\hline
\end{tabular}


annuelle sous forme de bois de tronc représentent entre 1,3 et 1,5 fois cette quantité sur l'ensemble de la révolution. Calculée à l'échelle du bassin versant, en incluant donc le peuplement de crête, moins productif, l'immobilisation est légèrement inférieure (tableau IV).

Les exportations sous forme de branches représentent environ $8,7 \mathrm{~kg} \mathrm{ha}^{-1} \mathrm{an}^{-1} \mathrm{~d}$ 'N, $0,8 \mathrm{~kg} \mathrm{ha}^{-1} \mathrm{an}^{-1}$ de P, $3,7 \mathrm{~kg} \mathrm{ha}^{-1} \mathrm{an}^{-1}$ de $\mathrm{K}$ et Ca et $1,8 \mathrm{~kg} \mathrm{ha}^{-1} \mathrm{an}^{-1}$ de $\mathrm{Mg}$. Au total, les exportations d'éléments minéraux par la récolte représentent, suivant le mode d'exploitation actuel, environ $13 \mathrm{~kg} \mathrm{ha}^{-1} \mathrm{an}^{-1} \mathrm{~d}$ 'N, $1 \mathrm{~kg} \mathrm{ha}^{-1} \mathrm{an}^{-1}$ de $\mathrm{P}, 8 \mathrm{~kg} \mathrm{ha}^{-1}$ $\mathrm{an}^{-1}$ de $\mathrm{K}$ et $\mathrm{Ca}$, et $4 \mathrm{~kg} \mathrm{ha}^{-1} \mathrm{an}^{-1}$ de $\mathrm{Mg}$. Avec l'exploitation des litières, l'exportation atteignait alors $32 \mathrm{~kg} \mathrm{ha}^{-1}$ $\mathrm{an}^{-1} \mathrm{~d}$ 'N, $2 \mathrm{~kg} \mathrm{ha}^{-1} \mathrm{an}^{-1}$ de P, $11 \mathrm{~kg} \mathrm{ha}^{-1} \mathrm{an}^{-1}$ de K, $18 \mathrm{~kg}$ $\mathrm{ha}^{-1} \mathrm{an}^{-1}$ de Ca, et $8 \mathrm{~kg} \mathrm{ha}^{-1} \mathrm{an}^{-1}$ de $\mathrm{Mg}$.

\subsection{Production et immobilisation forestière dans les bassins versants de Castrove et Jabali}

Les données dendrométriques des peuplements du bassin de Castrove sont présentées sur le tableau III. Suivant les tables de production galiciennes et en fonction de la hauteur dominante, le peuplement de la zone basse correspond à une classe II, celui du versant à une classe III, tandis que celui de la partie haute apparaît en classe V. Pour les peuplements de la zone basse et du versant, on peut estimer le volume exportable à $343 \mathrm{~m}^{3}$ et $216 \mathrm{~m}^{3} \mathrm{ha}^{-1}$ respectivement. Étant donné la très faible densité de la zone haute, nous avons négligé sa production. On calcule ainsi une production moyenne de bois sur l'ensemble du bassin versant et sur 15 ans de $11,8 \mathrm{~m}^{3}$ $\mathrm{ha}^{-1} \mathrm{an}^{-1}$. Sachant que l'infradensité moyenne du bois d'Eucalyptus globulus est de 0,58, que celle de l'écorce est de 0,38 , et en considérant un volume sous écorce égal à $83 \%$ du volume sur écorce [43], la biomasse récoltable correspond à $6,8 \mathrm{t} \mathrm{ha}^{-1} \mathrm{an}^{-1}$ de bois et $0,9 \mathrm{t} \mathrm{ha}^{-1} \mathrm{an}^{-1}$. d'écorce. Calculée sur la révolution de 15 ans, l'immobilisation moyenne annuelle de $\mathrm{N}, \mathrm{P}, \mathrm{K}, \mathrm{Ca}$ et $\mathrm{Mg}$ est approximativement de $7,6,6,8$ et $4 \mathrm{~kg} \mathrm{ha}^{-1} \mathrm{an}^{-1}$, dont entre $30 \%(\mathrm{~N})$ et $80 \%(\mathrm{Ca})$ dans l'écorce (tableau IV).

Pour le bassin versant de Jabali, dont les versants couvrent la majeure partie de la surface, nous avons fait l'hypothèse que les peuplements ont les mêmes caractéristiques que celui du versant de Castrove. On calcule alors une immobilisation annuelle légèrement supérieure $(20 \%)$ à celle du bassin de Castrove.

Tableau IV. Concentration en éléments minéraux de différents organes (\%), immobilisation moyenne annuelle de Matière Sèche $\left(\mathrm{t} \mathrm{ha}^{-1} \mathrm{an}^{-1}\right)$ et d'éléments minéraux $\left(\mathrm{kg} \mathrm{ha}^{-1} \mathrm{an}^{-1}\right)$ dans les bassins versants de Castrove, Jabali et Arcos.

\begin{tabular}{|c|c|c|c|c|c|c|c|c|}
\hline Bassin & & Compartiment & M. S. & $\mathrm{N}$ & $\mathrm{P}$ & $\mathrm{K}$ & $\mathrm{Ca}$ & $\mathrm{Mg}$ \\
\hline \multirow[t]{5}{*}{ Castrove } & Concentration & Ecorce & & 0,26 & 0,55 & 0,34 & 0,7 & 0,3 \\
\hline & & Bois & & 0,07 & 0,02 & 0,04 & 0,02 & 0,01 \\
\hline & Immobilisation moyenne & Ecorce & 0,9 & 2,3 & 5,0 & 3,1 & 6,3 & 2,7 \\
\hline & & Bois & 6,9 & 4,55 & 1,4 & 2,8 & 1,7 & 1,0 \\
\hline & & Ecorce + bois & 7,8 & 6,86 & 6,4 & 5,9 & 8,0 & 3,7 \\
\hline Jabali & Immobilisation moyenne & Ecorce + bois & 9,4 & 8,3 & 7,7 & 7,2 & 9,7 & 4,5 \\
\hline \multirow[t]{12}{*}{$\operatorname{Arcos}$} & Concentration & Ecorce & & 0,24 & 0,03 & 0,18 & 0,16 & 0,09 \\
\hline & & Bois & & 0,05 & 0,00 & 0,06 & 0,06 & 0,03 \\
\hline & & Branche & & 0,47 & 0,04 & 0,2 & 0,2 & 0,1 \\
\hline & & Aiguille & & 1,15 & 0,07 & 0,33 & 0,16 & 0,13 \\
\hline & & Litière & & 0,7 & 0,03 & 0,1 & 0,37 & 0,16 \\
\hline & Immobilisation moyenne & Bois+écorce & 4,5 & 3,0 & 0,3 & 3,2 & 3,1 & 1,5 \\
\hline & Exploitation d'intensité moyenne & Bois sur pied + éclaircies modérées & 6,0 & 4,0 & 0,4 & 4,3 & 4,1 & 2,0 \\
\hline & & Bois sur pied + éclaircies fortes & 6,6 & 4,4 & 0,4 & 4,7 & 4,5 & 2,2 \\
\hline & & Branche & 1,8 & 8,7 & 0,8 & 3,7 & 3,7 & 1,8 \\
\hline & & Litière & 2,7 & 18,9 & 0,8 & 2,6 & 9,9 & 4,3 \\
\hline & $\begin{array}{l}\text { Exploitation actuelle } \\
\text { (éclaircies moyennes) }\end{array}$ & Tous compartiments & 7,9 & 12,7 & 1,2 & 8,0 & 7,8 & 3,8 \\
\hline & Exploitation traditionnelle & Tous compartiments & 10,6 & 31,6 & 2,0 & 10,6 & 17,7 & 8,1 \\
\hline
\end{tabular}




\subsection{Bilans hydrologiques (tableau $V$ )}

Les précipitations cumulées entre le 29/4/94 et le 26/4/95 s'élèvent à $2245 \mathrm{~mm}$ à Castrove et $1622 \mathrm{~mm}$ à Arcos, valeurs légèrement supérieures à la moyenne mesurée au cours des 7 années précédentes. Le drainage s'élève à Castrove à $1555 \mathrm{~mm}$ et à $1533 \mathrm{~mm}$ à Jabali. La différence, correspondant à l'évapo-transpiration réelle (ETR), s'éleve à $690 \mathrm{~mm}$ à Castrove et $712 \mathrm{~mm}$ à Jabali, des valeurs sensiblement inférieures à l'évapo-transpiration potentielle (ETP) sur la même période (1100 mm). Ces faibles valeurs d'ETR proviennent de ce que la couverture forestière des bassins versants n'est que partielle, suite aux coupes rases du bassin de Castrove en 1992, et aux coupes partielles du bassin versant de Jabali. Par comparaison, la différence (précipitation - drainage) à Castrove s'élevait à $1061 \mathrm{~mm}$ pendant l'année hydrologique 1987-88, avant les incendies, et elle avait décru à 352 mm en 1992-1993, après la coupe rase [20].

Le bilan du $\mathrm{Cl}$ est équilibré à Castrove ce qui confirme le bilan hydrologique. A Jabali par contre, les sorties des $\mathrm{Cl}\left(175 \mathrm{~kg} \mathrm{ha}^{-1} \mathrm{an}^{-1}\right)$ dépassent de $30 \%$ les entrées par les pluies $\left(135 \mathrm{~kg} \mathrm{ha}^{-1} \mathrm{an}^{-1}\right)$. Cette différence peut être au moins partiellement attribuée à des apports sous formes de brouillards d'advection, très fréquents sur ces premiers reliefs face à l'océan. Ainsi Gras [20], en comparant les volumes de pluies et pluviolessivats recueillis pendant des périodes avec ou sans brouillard, a mesuré dans des peuplements d'eucalyptus adultes du bassin versant, des interceptions positives proches de $14 \%$ des précipitations hors couvert pendant les périodes sans brouillards, et des interceptions négatives de l'ordre de $-7 \%$ pendant les périodes de brouillard. Cet effet d'interception des brouillards par les houppiers est certainement beaucoup plus faible à Castrove, en raison de la jeunesse du peuplement.

À Arcos, sur la même période, le drainage annuel est de $493 \mathrm{~mm}$ pour une hauteur de précipitation de $1622 \mathrm{~mm}$. La différence, $1128 \mathrm{~mm}$, est proche de la valeur de l'ETP et des bilans hydrologiques enregistrés dans ce bassin versant depuis 1987. Le bilan du Cl est proche de l'équilibre. L'importance des dépôts par les brouillards dans ce bassin versant situé à $5 \mathrm{~km}$ de la mer,

Tableau V. Bilan annuel d'éléments minéraux apportés par les précipitations, drainés par le ruisseau et exportés dans la biomasse forestière.

\begin{tabular}{|c|c|c|c|c|c|c|c|c|c|}
\hline & eau & $\mathrm{Na}$ & K & $\mathrm{Mg}$ & $\mathrm{Ca}$ & $\mathrm{Cl}$ & $\mathrm{N}-\mathrm{NO}_{3}$ & $\mathrm{~S}-\mathrm{SO}_{4}$ & $\mathrm{Si}$ \\
\hline & $\mathrm{mm}$ & \multicolumn{7}{|c|}{$\mathrm{kg} \mathrm{ha}^{-1} \mathrm{yr}^{-1}$} & \\
\hline \multicolumn{10}{|l|}{ Castrove } \\
\hline Precipitation & 2245 & 69,7 & 4,1 & 9 & 5,3 & 135,1 & 1 & 13,8 & \\
\hline Drainage & 1555 & 78,4 & 1,7 & 7,9 & 2,9 & 135 & 0,3 & 9 & 31,8 \\
\hline P-D & 690 & $-8,7$ & 2,4 & 1,1 & 2,4 & 0,1 & 0,7 & 4,8 & $-31,8$ \\
\hline Exploitation forestière & & & 5,9 & 3,6 & 7,9 & & & & \\
\hline P-D-E & & & $-3,5$ & $-2,6$ & $-5,6$ & & & & \\
\hline \multicolumn{10}{|l|}{ Jabali } \\
\hline Precipitation & 2245 & 69,7 & 4,1 & 9 & 5,3 & 135,1 & 1 & 13,8 & \\
\hline Drainage & 1533 & 96,3 & 3,5 & 10,2 & 4,8 & 174,8 & 1 & 9,4 & 39,8 \\
\hline P-D & 712 & $-26,6$ & 0,6 & $-1,3$ & 0,5 & $-39,7$ & & 4,3 & $-39,8$ \\
\hline \multicolumn{10}{|l|}{ Precipitation corrigée* } \\
\hline $\begin{array}{l}(\mathrm{Cl} \text { entrée }=\mathrm{Cl} \text { sortie }) \\
\mathrm{P}^{*} \text {-D }\end{array}$ & & $\begin{array}{l}90,2 \\
-61\end{array}$ & $\begin{array}{l}5,3 \\
18\end{array}$ & 11,6 & $\begin{array}{l}6,9 \\
2,1\end{array}$ & $\begin{array}{c}174,8 \\
0\end{array}$ & 1,3 & $\begin{array}{l}17,8 \\
8,4\end{array}$ & \\
\hline $\begin{array}{l}\mathrm{P}^{*}-\mathrm{D} \\
\text { Exploitation forestière }\end{array}$ & & & $\begin{array}{l}1,0 \\
7,2\end{array}$ & $\begin{array}{l}1,5 \\
4,5\end{array}$ & 9,7 & & 0,3 & 8,4 & $-39,8$ \\
\hline $\begin{array}{l}\text { Exploitation forestière } \\
\text { P*-D-E }^{*}\end{array}$ & & & $-5,3$ & $-3,1$ & $-7,5$ & & & & \\
\hline \multicolumn{10}{|l|}{ Arcos } \\
\hline Precipitation & 1622 & 32,2 & 2,5 & 4,3 & 2,8 & 62,6 & 0,4 & 8,0 & \\
\hline Drainage & 493 & 36,8 & 0,7 & 3,1 & 2,6 & 59,6 & 0,0 & 2,7 & 24,6 \\
\hline P-D & 1128 & $-4,6$ & 1,7 & 1,1 & 0,1 & 3 & 0,4 & 5,3 & $-24,6$ \\
\hline Exploitation actuelle & & 0,6 & 8,0 & 3,7 & 7,8 & & & 2,3 & \\
\hline Exploitation traditionnelle & & & 10,5 & 8,0 & 17,7 & & & 4,7 & \\
\hline P-D-E actuelle & & & $-6,3$ & $-2,6$ & $-7,7$ & 3 & & 2,9 & \\
\hline P-D-E traditionnelle & & & $-8,8$ & $-6,9$ & $-17,6$ & 3 & & 0,5 & \\
\hline
\end{tabular}


derrière une première barrière orographique et à faible altitude est bien moindre qu'à Jabali.

\subsection{Concentrations en solution et bilans hydrochimiques}

Les précipitations sont très diluées, très peu chargées en polluants atmosphériques ( $\mathrm{S}$ et $\mathrm{N}$ ) et neutres (tableau VI). La composition des précipitations reflète fortement celle de l'eau de mer : les rapports molaires $\mathrm{Na} / \mathrm{Cl}, \mathrm{Mg} / \mathrm{Cl}, \mathrm{SO}_{4} / \mathrm{Cl}$ à Castrove sont respectivement égaux à $0,8,0,2$ et 0,11 tandis que ces mêmes rapports dans l'eau de mer valent $0,86,0,2$ et 0,10 . Par contre, les précipitations sont enrichies en $\mathrm{Ca}$ et $\mathrm{K}$ par rapport à l'eau de mer. L'influence océanique est beaucoup plus forte à Castrove, tandis que l'influence continentale se fait légèrement plus sentir à Arcos, à plus grande distance de la mer. Les précipitations déposent moins de $2 \mathrm{~kg}$ $\mathrm{ha}^{-1} \mathrm{an}^{-1}$ d'azote nitrique, entre 8 et $14 \mathrm{~kg} \mathrm{ha}^{-1} \mathrm{an}^{-1}$ de soufre essentiellement d'origine marine, et $5 \mathrm{~kg} \mathrm{ha}^{-1} \mathrm{an}^{-1}$ de calcium à Castrove. Les précipitations sont moins abondantes à Arcos de sorte qu'elles engendrent des dépôts environ $40 \%$ plus faibles qu'à Castrove.

Les eaux des ruisseaux ont une composition très proche de celle des précipitations (tableau VI). La seule différence notable concerne les concentrations en $\mathrm{Na}$ et $\mathrm{Cl}$, qui sont environ 2 fois plus élevées dans les ruisseaux que dans les pluies et la teneur en Si. pH et concentrations moyennes en $\mathrm{Ca}, \mathrm{Mg}, \mathrm{K}$ et $\mathrm{Na}$ sont plus faibles à l'exutoire du bassin versant de Castrove qu'à l'exutoire du bassin de Jabali, ou dans les eaux du ruisseau drainant le bassin versant d'Arcos. Par contre, le drainage d'azote nitrique est le plus élevé à Jabali, et le plus faible à Arcos.
L'évolution des concentrations en $\mathrm{Ca}, \mathrm{Mg}, \mathrm{K}, \mathrm{Na}$ et $\mathrm{NO}_{3}$ entre 1993 et 1995, montre une baisse continue des concentrations en $\mathrm{K}$ et $\mathrm{NO}_{3}$, qui témoigne de l'arrière effet des coupes dans ces trois bassins versants (figure 2). Par rapport aux concentrations mesurées en 1987 à Castrove et Arcos, avant les incendies et les coupes [21], les concentrations moyennes en $\mathrm{Ca}$, $\mathrm{K}$ et $\mathrm{NO}_{3}$ pendant l'année 1994-95 demeurent légèrement plus élevées. Cependant la différence de concentration résulte au moins en partie de ce que les précipitations en 1987 (3300 mm à Castrove) ont été près de $50 \%$ supérieures aux précipitations moyennes et à celles de 1995, de sorte que l'on peut considérer les flux mesurés en 1995 comme proches de l'équilibre.

Les bilans hydrochimiques des trois bassins versants calculés sur la période 1994-1995 sont présentés sur le tableau $V$. À Castrove, le bilan précipitation-drainage $(\mathrm{P}-\mathrm{D})$ indique des pertes en $\mathrm{Na}$ et $\mathrm{Si}$ de 8,7 et $31,8 \mathrm{~kg}$ $\mathrm{ha}^{-1} \mathrm{an}^{-1}$, et des gains de $\mathrm{Ca}\left(2,5 \mathrm{~kg} \mathrm{ha}^{-1} \mathrm{an}^{-1}\right), \mathrm{Mg}$ $\left(1,1 \mathrm{~kg} \mathrm{ha}^{-1} \mathrm{an}^{-1}\right), \mathrm{K}\left(2,5 \mathrm{~kg} \mathrm{ha}^{-1} \mathrm{an}^{-1}\right), \mathrm{N}\left(3,2 \mathrm{~kg} \mathrm{ha}^{-1}\right.$ $\left.\mathrm{an}^{-1}\right)$ et $\mathrm{S}\left(4,8 \mathrm{~kg} \mathrm{ha}^{-1} \mathrm{an}^{-1}\right)$. À Jabali, pour tenir compte des dépôts par les brouillards, nous avons corrigé les valeurs de dépôts en les multipliant par le rapport des flux $\mathrm{Cl}$ ruisseau/Cl pluie. On obtient alors des gains et pertes proches de ceux calculés à Castrove. A Arcos, les pertes de $\mathrm{Na}$ et $\mathrm{Si}$ sont plus faibles, respectivement $4,6 \mathrm{~kg} \mathrm{ha}^{-1} \mathrm{an}^{-1}$ et $24,7 \mathrm{~kg} \mathrm{ha}^{-1} \mathrm{an}^{-1}$, les gains en $\mathrm{K}$ et $\mathrm{Mg}$ sont proches de ceux calculés dans les autres bassins, tandis que le bilan du Ca est équilibré.

Globalement, les sols fournissent aux ruisseaux entre 5 et $9 \mathrm{~kg} \mathrm{ha}^{-1} \mathrm{an}^{-1}$ de $\mathrm{Na}$ et 25 à $40 \mathrm{~kg} \mathrm{ha}^{-1} \mathrm{an}^{-1}$ de $\mathrm{Si}$, tandis que 0 à $3 \mathrm{~kg} \mathrm{ha}^{-1} \mathrm{an}^{-1}$ de Ca, $1 \mathrm{~kg} \mathrm{ha}^{-1} \mathrm{an}^{-1}$ de $\mathrm{Mg}, 2$ à $3 \mathrm{~kg} \mathrm{ha}^{-1} \mathrm{an}^{-1} \mathrm{~d}^{\prime} \mathrm{N}, 5$ à $8 \mathrm{~kg} \mathrm{ha}^{-1} \mathrm{an}^{-1}$ de $\mathrm{S}$ et 2 à $3 \mathrm{~kg} \mathrm{ha}^{-1} \mathrm{an}^{-1}$ de $\mathrm{K}$ sont retenus dans l'écosystème.

Tableau VI. Composition chimique des précipitations et eaux de ruisseau et comparaison des rapports molaires de concentration avec l'eau de mer.

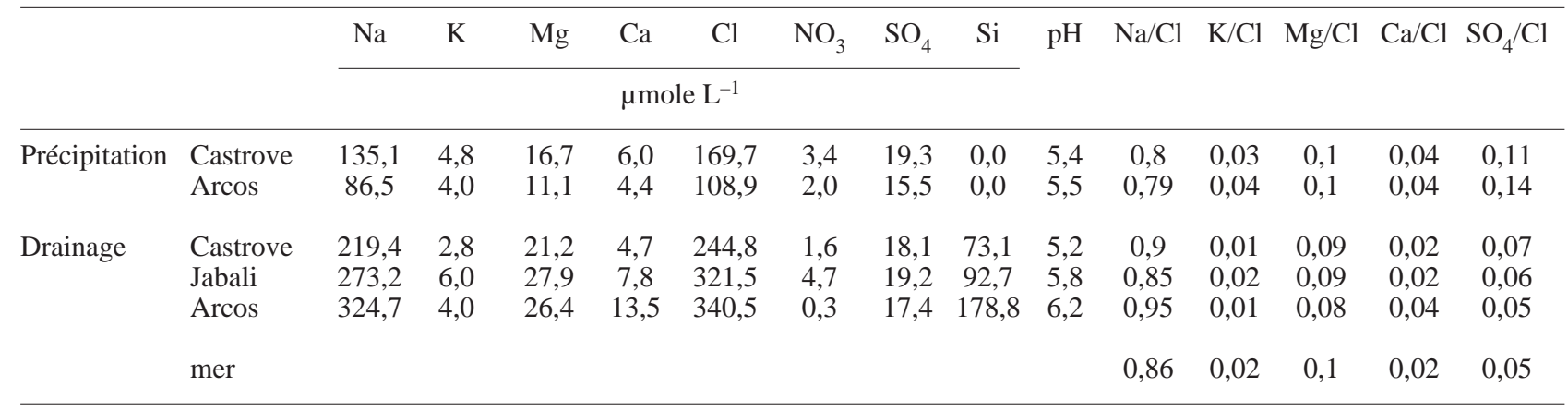




\section{CASTROVE}

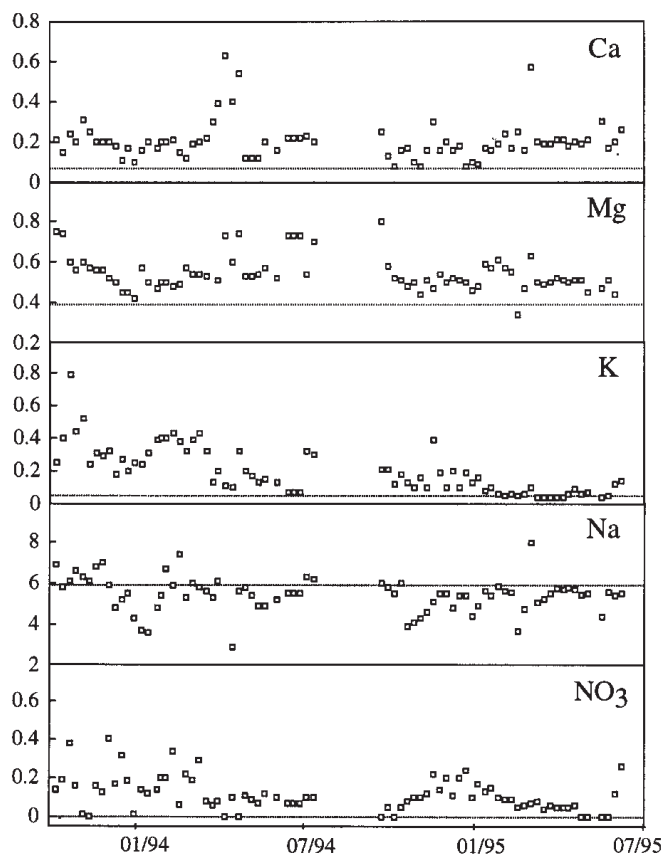

$\operatorname{ARCOS}$

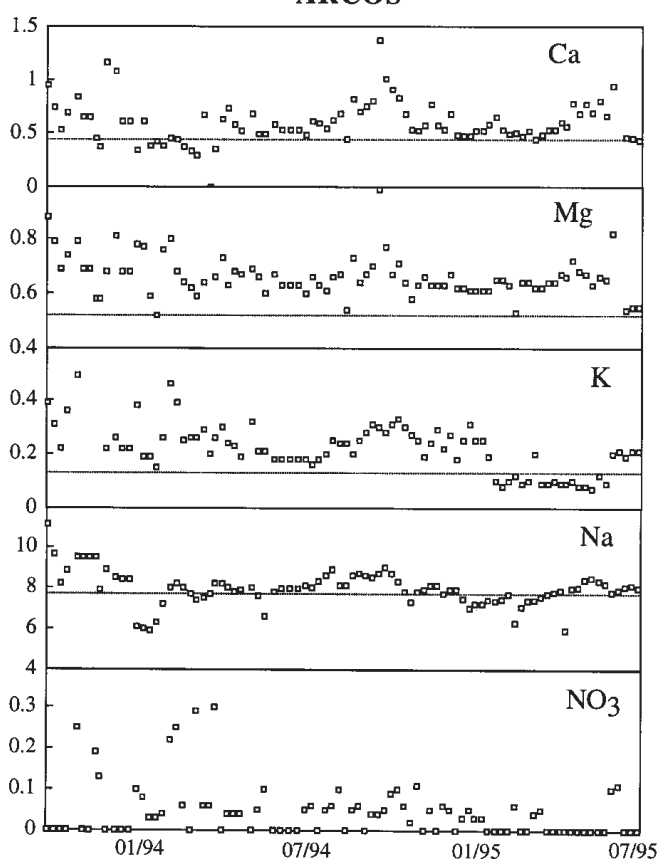

Figure 2. Évolution temporelle des concentrations en $\mathrm{Ca}, \mathrm{Mg}$, $\mathrm{K}, \mathrm{Na}$ et $\mathrm{NO}_{3}\left(\mathrm{mg} \mathrm{L}^{-1}\right)$ des ruisseaux des bassins versants de Castrove et Jabali en 1994-95. Le niveau des concentrations moyennes annuelles mesurées en 1987, avant les coupes forestières et les incendies, est indiqué sur chaque graphe par une ligne pointillée horizontale.

\section{DISCUSSION GÉNÉRALE}

\subsection{Propriétés des sols}

La distribution des sols observée dans ces basssins versants est classique en Galice, le climat doux et pluvieux favorisant une altération minérale rapide, libérant une grande quantité d'Al amorphe à même de séquestrer la matière organique et de favoriser son accumulation. La présence dans les combes et les replats, de sols organiques à caractère cumullique a été décrite et analysée par Guitian Ojea and Carballas [24]. Dans les horizons dépourvus de matière organique se forment des argiles 1:1 (kaolinite, halloisite) et de la gibbsite [33]. La formation de gibbsite est favorisée à Jabali par la forte pluviométrie, la pente accentuée, la faible teneur en cations alcalino-terreux des minéraux et la perméabilité du matériel sableux issu de la désagrégation du granite. Pour des raisons inverses, la formation de kaolinite domine à Arcos. A l'extrême, en bas de pente, dans la zone de battement de nappe, des conditions de relatif confinement apparaissent et donnent lieu à la synthèse de smectite $[34,42]$. Dans ce contexte textural, la capacité d'échange des sols dépend en majeure partie de la matière organique. Les cations alcalins et alcalino-terreux libérés par l'altération sont peu retenus par la faible capacité d'échange des argiles, et en conséquence drainés hors des profils. Les sols sont profondément désaturés : les valeurs de $\mathrm{Ca}$ et $\mathrm{Mg}$ échangeables sont nulles sur granite, et à peine plus élevées sur granodiorite. Ces valeurs ne s'élèvent légèrement que dans l'horizon organique, par recyclage biologique, et dans les zones de confinement ou s'accumule le produit du drainage des versants.

\subsection{Bilans à l'échelle des bassins versants}

Les bassins versants étudiés se caractérisent par des flux entrant de $\mathrm{Ca}, \mathrm{Mg} \mathrm{K}$ et $\mathrm{N}$ de même ordre de grandeur ou plus élevés que les flux sortants dans les eaux de drainage. Ce fait pourrait être imputable à des erreurs de mesure du bilan hydrologique liées à la brieveté de la période de mesure dans la cas de Jabali mais les concentrations des eaux mesurées au cours du cycle 94-95 sont encore marquées par la minéralisation associée au feu et à la coupe rase (figure 2). Mais cette particularité est fréquente en Galice. L'analyse chimique d'eaux issues d'une trentaine de bassins versants Galiciens sur granite nous a confirmé les très faibles concentrations en cations alcalins et alcalino-terreux mesurées en continu dans les bassins de Castrove et Jabali (Garcia Rodeja et Dambrine 1995, données non publiées). Issu d'une revue de la littérature, le tableau VII présente les termes de bilans hydrochimiques (pluie - drainage) mesurés pour une gamme 


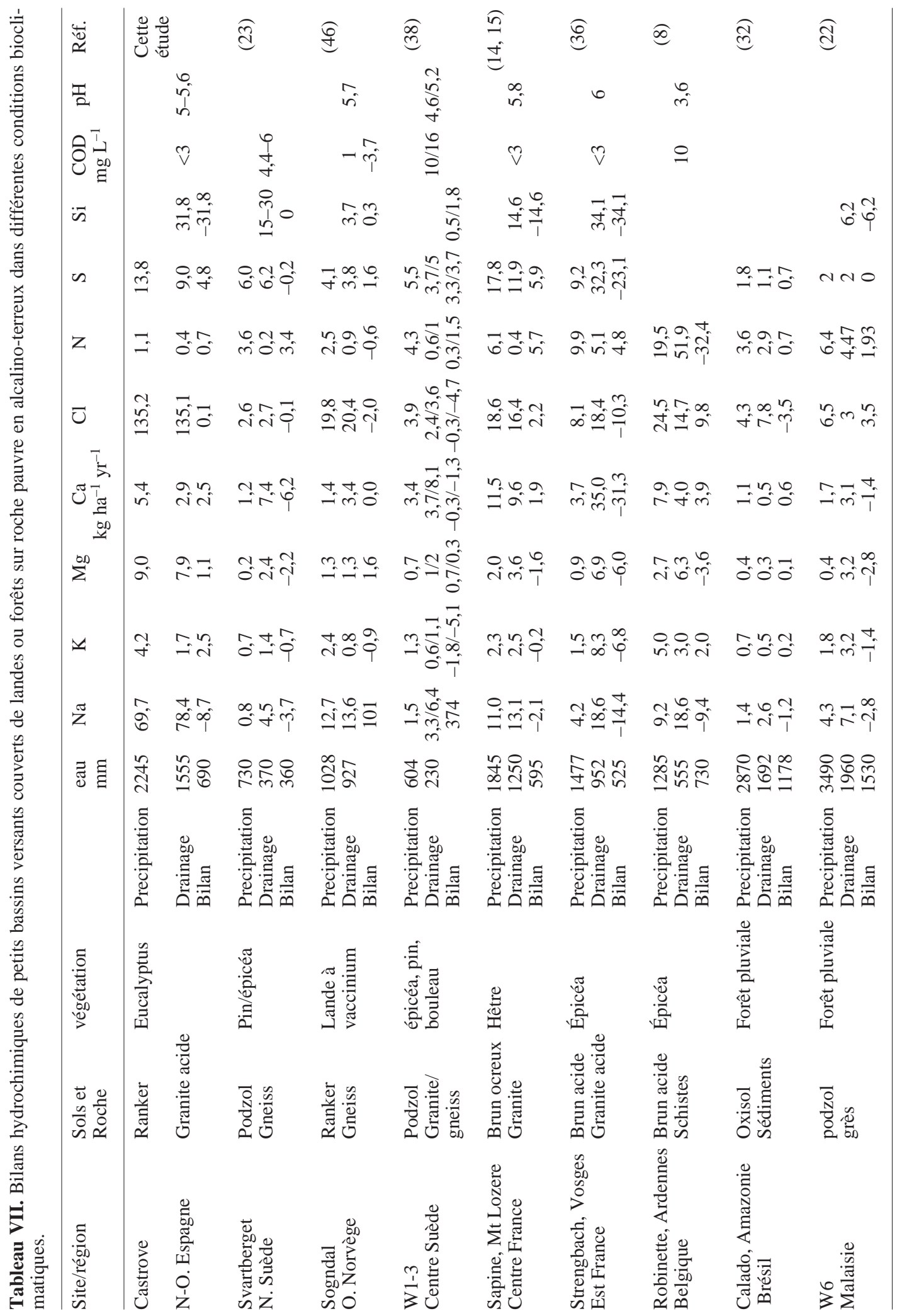


de climats variés dans des bassins versants sur roche pauvre en minéraux altérables, diversement affectés par la pollution atmosphérique. Nous avons particulièrement sélectionné les bilans positifs.

L'azote, en dehors de conditions extrêmes de dépôt ou de déboisement est généralement retenu dans les bassins versants quelque soient les conditions climatiques. Par contre, les bilans (pluie - drainage) de $\mathrm{Ca}, \mathrm{Mg}$ et $\mathrm{K}$ positifs à l'échelle du bassin versant sont très rares. $\mathrm{Ca}, \mathrm{Mg}$ et $\mathrm{K}$ sont perdus en quantités variables dans les bassins versants sur gneiss sous climat boréal de Svartberget [23] et Sogndal [46], pratiquement indemnes de pollution. Dans le premier cas, il s'agit d'un bassin versant forestier relativement productif mais dont les eaux sont chargées en acides organiques et drainent un gneiss relativement riche en minéraux altérables. Dans le second, la croissance de la végétation comme la récolte de biomasse est extrêmement faible. Dans une comparaison de trois bassins forestiers productifs sur leucogranite du centre de la Suède, Rosen [38] montre que le seul bassin versant dont le bilan de Ca soit proche de l'équilibre se caractérise par une plus faible teneur en acides organiques dissous. Les bilans négatifs de $\mathrm{Ca}, \mathrm{Mg}$ et $\mathrm{K}$ sont généralisés dans l'ensemble des petits bassins forestiers expérimentaux sur roche pauvre en minéraux altérables d'Europe occidentale [26] et des États-Unis [3] diversement affectés par la pollution atmosphérique et dont les eaux contiennent peu de carbone organique dissous. L'exemple du bilan négatif du bassin versant forestier du Strengbach [36], sur un granite extrêmement pauvre en $\mathrm{Ca}$ et $\mathrm{Mg}$, illustre cette tendance. Quelques bassins font cependant exception. La hêtraie peu productive du Mont Lozère, dont les sols sont peu épais et riches en matière organique, reçoit de forts apports de $\mathrm{Ca}$ et de $\mathrm{S}$ et les accumule $[14,15]$. Une autre exception nous est fournie par le bassin versant résineux et productif de la Robinette (Ardennes), dont les sols peu épais, reposant sur des schistes imperméables et très pauvres en alcalino-terreux, accumulent le $\mathrm{Ca}$ [8]. Les bilans de $\mathrm{K}$ et $\mathrm{Mg}$ de ces deux derniers bassins sont cependant négatifs. Dans une étude effectuée sur de grands bassins versants forestiers chiliens sous climat océanique tempéré (proche de celui de la Galice), ne subissant ni pollution ni exploitation forestière, Hedin et al. [25], indiquent des pertes en Ca systématiques, et de faibles pertes en $\mathrm{Mg}$, tandis que les bilans de $\mathrm{Na}$ et $\mathrm{K}$ sont équilibrés. Sous forêt tropicale non perturbée, Lesak and Melak [32] mesurent des bilans positifs en $\mathrm{Ca}, \mathrm{Mg}, \mathrm{K}, \mathrm{N}$ et $\mathrm{S}$ dans un bassin d'Amazonie centrale couvert d'oxisols. De tels gains ont été précédemment décrits à l'échelle du sol par Jordan (28) comme une caractéristique des écosystèmes amazoniens. Par contre, en Malaisie, Grip et al. [22] mesurent des pertes en $\mathrm{Ca}, \mathrm{Mg}$ et $\mathrm{K}$ et des gains d'N dans des bassins versants à podzols, avec une forte charge organique dans le ruisseau.

La combinaison de plusieurs facteurs explique le signe de ces bilans. Tout d'abord, la charge cationique des eaux de surface est liée à leur charge anionique. La pollution atmosphérique azotée et soufrée, largement répandue en Europe du Nord et de l'Est, augmente la charge en anions $\left(\mathrm{SO}_{4}\right.$ et $\left.\mathrm{NO}_{3}\right)$ des ruisseaux et par conséquence, la charge en cations. Les podzols et tourbières développés sous climat boréal ou tropical sont riches en anions organiques solubles et leur capacité d'adsorption du sulfate est limitée. Ensuite, les sols développés sous des climats humides océaniques ou (sub)tropicaux sont plus profondément altérés et donc appauvris en cations alcalino-terreux que les sols tempérés. Leur potentiel de libération de cations par altération est donc réduit et par ailleurs leur capacité d'adsorption du sulfate est généralement élevée. Enfin, la végétation prélève des ions qui, sinon, pourraient alimenter le débit chimique du ruisseau, et ceci d'autant plus que sa biomasse s'accroît et qu'elle est exportée. Les bilans positifs mesurés en Galice reflètent donc la combinaison : roche et sols pauvres en cations alcalino-terreux + carbone immobile + pollution nulle + forte immobilisation forestière.

En dépit de leur forte croissance, les peuplements d'eucalyptus des bassins versants de Castrove et Jabali s'avèrent relativement frugaux, du fait essentiellement des très faibles concentrations mesurées dans le bois [30, 31]. Si l'on compare les gains calculés par le bilan hydrochimique, et l'immobilisation forestière, il vient que le bilan serait approximativement équilibré si seul le bois était exporté, sauf pour l'azote. L'exportation de l'écorce déséquilibre nettement le bilan. Le bilan actuel à Castrove indique des pertes annuelles par hectare en $\mathrm{Ca}$, $\mathrm{Mg}, \mathrm{K}$ et $\mathrm{N}$ respectivement égales à $5,5 \mathrm{~kg}, 2,6 \mathrm{~kg}$, $3,5 \mathrm{~kg}$, et $6,1 \mathrm{~kg}$. Des pertes environ $50 \%$ plus élevées sont mesurées à Jabali. Une partie de ces pertes peut être attribuée à une minéralisation excessive dans les sols, liée à la coupe précédente. Si l'on calcule ces bilans à Castrove en utilisant les concentrations moyennes mesurées en 1987, c'est-à-dire avant les incendies et la coupe, les pertes se réduisent à environ $4 \mathrm{~kg}$ de $\mathrm{Ca}, 2,3 \mathrm{~kg}$ de $\mathrm{Mg}, 2,5 \mathrm{~kg}$ de $\mathrm{K}$ et $5,7 \mathrm{~kg}$ d'N. Cependant les concentrations élevées mesurées dans le ruisseau pendant les années qui ont suivi les incendies et coupes montrent que les pertes ont alors été bien supérieures aux pertes actuelles. Les valeurs de 1994-95 s'approchent donc plus probablement des pertes moyennes sur la révolution que celles de 1987.

À Arcos, les entrées par les pluies étant peu différentes des sorties dans le ruisseau, le mode d'exploitation de la forêt modifie profondément les termes du bilan. 
L'exploitation actuelle des grumes et des branches conduit à des pertes annuelles par hectare en $\mathrm{Ca}, \mathrm{Mg}, \mathrm{K}$ et $\mathrm{N}$ respectivement égales à $7,8 \mathrm{~kg}, 2,7 \mathrm{~kg}, 6,4 \mathrm{~kg}$, et $2,9 \mathrm{~kg} \mathrm{ha}^{-1} \mathrm{an}^{-1}$, l'exploitation des branches contribuant environ pour la moitié de ces valeurs. Suivant les hypothèses faites, l'exploitation traditionnelle des litières creuse considérablement le déficit du bilan.

\section{3. Évolution de la fertilité minérale des sols}

Pour évaluer l'acidification des sols induite par ces pratiques forestières, des bilans minéraux doivent être effectués à l'échelle du sol et plus particulièrement du pool d'éléments assimilables à court terme par la forêt, stocké sous forme échangeable ou organique dans le sol. Pour cela, deux termes supplémentaires doivent être estimés : l'altération minérale, et l'accumulation ou la minéralisation de composés organiques.

L'accumulation organique n'a été mesurée dans aucun des bassins versants. À Jabali, on peut penser que la phase de développement du peuplement d'eucalyptus correspond à une phase d'accumulation de matière organique sous forme de branches, et sous forme de litière à la surface du sol. En effet, si les retombées de litières sous forme de feuilles sont rapidement minéralisée, il en va autrement des écorces qui peuvent représenter une part importante des chutes de litière, se dégradent plus lentement et contiennent des teneurs élevées en éléments minéraux. À Castrove, les incendies ont accéléré la minéralisation des litières. Les flux d'azote nitrique dans le ruisseau témoigneraient de cette minéralisation (figure 2).

À Arcos, la pinède est relativement ancienne, le bassin versant ne perd pas d'azote mais la récolte de matière organique a décru en intensité depuis 20 années de sorte que l'on peut faire l'hypothèse d'un état stable ou d'une légère accumulation organique.

Pour les deux bassins d'Arcos et de Jabali, on peut faire l'hypothèse d'une stabilité ou d'une légère augmentation du compartiment organique.

L'altération n'est pas mesurable directement. À Castrove et Jabali, on pourrait considérer que sa valeur nous est fournie par le bilan (entrées - sorties) car le taux de saturation des sols en cations alcalins et alcalino-terreux est tellement bas qu'il ne peut guère s'abaisser encore. Cependant, on pourrait concevoir que la teneur en éléments nutritifs des eucalyptus (bien qu'elle soit déjà très basse) s'abaisse progressivement au cours des prochaines rotations. Cette hypothèse conduit à des flux maximum de $\mathrm{Ca}, \mathrm{Mg}, \mathrm{K}$ et $\mathrm{Na}$ libérés par altération de l'ordre de 5 à $7 \mathrm{~kg}$ de $\mathrm{Ca}, 3 \mathrm{~kg}$ de $\mathrm{Mg}, 3$ à $5 \mathrm{~kg}$ de $\mathrm{K}$. Ce flux apparaît relativement élevé si l'on se réfère aux teneurs totales de la terre fine des sols en $\mathrm{Ca}$ et $\mathrm{Mg}$, proches de $0,1 \%$ (tableau I). Cependant, les éléments grossiers des sols, qui sont très abondants pourrait contribuer notablement à la nutrition minérale des peuplements [45]. Dans ces bassins versants, l'argile dominante est la gibbsite, que ce soit dans la fraction argileuse totale ou dans la fraction $<0,2 \mu$, tandis que la kaolinite est abondante dans la fraction limoneuse et argile grossière. On peut ainsi estimer que la silice est peu conservée dans le bassin, et qu'en conséquence l'exportation de $\mathrm{Si}$ dans le ruisseau représente pour l'essentiel le flux issu de l'altération des minéraux primaires.

En faisant l'hypothèse que la gibbsite est la seul argile synthétisée dans les sols, le bilan des alcalins et alcalinoterreux libérés par mole de Si dissoute se calcule alors en considérant [44] que, suivant sa nature, la dissolution d'un feldspath libère trois moles de $\mathrm{Si}$ par mole de $\mathrm{Na}$ ou $\mathrm{K}$ libéré, ou deux moles de Si par mole de Ca libéré, suivant les équations :

$$
\begin{aligned}
\mathrm{Si}_{3} \mathrm{AlO}_{8} \mathrm{~K} & +7 \mathrm{H}_{2} \mathrm{O}+\mathrm{H}_{2} \mathrm{CO}_{3} \rightarrow 3 \mathrm{H}_{4} \mathrm{SiO}_{4}+\mathrm{K}^{+} \\
& +\mathrm{Al}(\mathrm{OH})_{3}+\mathrm{HCO}_{3}^{-} \\
\mathrm{Si}_{2} \mathrm{Al}_{2} \mathrm{O}_{8} \mathrm{Ca} & +6 \mathrm{H}_{2} \mathrm{O}+2 \mathrm{H}_{2} \mathrm{CO}_{3} \rightarrow 2 \mathrm{H}_{4} \mathrm{SiO}_{4}+\mathrm{Ca}^{2+} \\
+ & 2 \mathrm{Al}(\mathrm{OH})_{3}+2 \mathrm{HCO}_{3}^{-}
\end{aligned}
$$

Le rapport K/Si étant le même dans une biotite et dans une orthose, le $\mathrm{Mg}$ éventuellement libéré par dissolution d'une biotite ne doit pas être compté.

$\mathrm{Si}$ le flux net de $\mathrm{Ca}, \mathrm{Mg}$ et $\mathrm{K}$ perdu par le bassin versant de Castrove provenait de l'altération de ces minéraux, cette dissolution libérerait un flux de Si égal à $1,65 \mathrm{kmoles} \mathrm{ha}^{-1} \mathrm{an}^{-1}$. Ces pertes sont très supérieures à celles mesurées $(1,14)$. Si l'on ne tient pas compte du Ca libéré, du fait que sa source la plus probable est l'apatite, dont la dissolution ne libère pas de $\mathrm{Si}$, les pertes attendues sont de 1,37 kmole ha ${ }^{-1} \mathrm{an}^{-1}$, valeur proche mais toujours supérieure aux pertes mesurées. À Jabali le calcul fournit un flux de Si potentiellement libéré $(1,56)$ proche de la valeur mesurée $(1,43)$. Si l'on considère que le $\mathrm{Ca}$ provient de l'apatite, on obtient un flux de $\mathrm{Si}$ de 1,18 , plus faible que celui mesuré. Les roches mères et cortèges minéralogiques des bassins de Castrove et Jabali étant semblables, il est peu probable que les néoformations diffèrent. Les fortes pertes en cations alcalins et alcalino-terreux par rapport à la silice à Castrove reflètent en partie le niveau de concentration relativement élevé en $\mathrm{K}$ du ruisseau, consécutif aux coupes. Le bon accord entre l'estimation et la mesure à Jabali suggère que l'altération est la source principale des cations alcalins. Le bilan du Ca pèse relativement peu (par rapport à $\mathrm{K})$ sur le résultat final de ce calcul, qui s'avère peu adapté pour mesurer la libération de Ca par altération. 
Un autre calcul peut être mené sur la base des bilans comparés de $\mathrm{Ca}$ et de $\mathrm{Na}$. Si l'on ne tient pas compte du Ca présent dans l'apatite, ces deux éléments sont les constituants des plagioclases, et sont donc libérés parallèlement au cours de la dissolution congruente de ce minéral, dans des proportions égales à celles du minéral. Le rapport $\mathrm{Ca} / \mathrm{Na}$ des échantillons de sols varie autour de $0,1 \mathrm{~g} \mathrm{~g}^{-1}$ à Castrove et Jabali. Si l'on considère que l'ensemble du Na libéré par altération est exporté par le ruisseau, il vient que l'altération des plagioclases libérerait moins de $0,5 \mathrm{~kg}$ de $\mathrm{Ca}$. Cette valeur est très inférieure au déficit du bilan, dont le comblement impliquerait donc une contribution importante de l'apatite. Des résultats similaires ont été obtenus par Fichter et al. [18] dans un contexte tempéré. Si l'on conserve comme hypothèse que l'altération compense intégralement le déficit du bilan et que l'on considère que les peuplements et le ruisseau s'alimentent à partir d'un réservoir homogène issu du mélange entre les apports par la pluie et l'altération, il découle que la pluie fournirait aux peuplement d'eucalyptus de Jabali respectivement environ $40 \%, 75 \%$ et $45 \%$ de leur alimentation en $\mathrm{Ca}, \mathrm{Mg}$ et $\mathrm{K}$. Ces proportions sont vraisemblablement sous-estimées car la contribution de l'altération des horizons profonds à la charge chimique du ruisseau est plus forte, tandis que les pluies alimentent plus probablement les horizons supérieurs ou se trouvent les racines [12]. Les pluies jouent donc un rôle capital dans l'alimentation de ces peuplements.

À Arcos, on peut raisonnablement faire l'hypothèse que la capacité d'échange des sols ne se désature pas actuellement, ou que les pertes annuelles de cations échangeables ne représentent qu'une faible fraction du stock de cations échangeables. En effet, le régime d'exploitation actuel de la forêt est beaucoup moins appauvrissant que ce qu'il a pu être dans le passé et le taux de saturation des sols n'est réellement très bas que sur la crête du bassin. On obtient alors un flux d'altération égal à : 7,8 $\mathrm{kg}$ de $\mathrm{Ca}, 2,7 \mathrm{~kg}$ de $\mathrm{Mg}, 6,4 \mathrm{~kg}$ de $\mathrm{K}$ et $4,6 \mathrm{~kg}$ de Na.

Dans ce bassin, les argiles néoformées sont la gibbsite et des minéraux 1:1 de la famille de la kaolinite. Si une kaolinite est néoformée, la dissolution d'un feldspath fournit deux moles de Si par mole de $\mathrm{Na}$ ou K libérée, la dissolution d'un plagioclase calcique ne fournissant pas de Si à la solution. Suivant que l'on considère que la gibbsite ou la kaolinite se forme, la dissolution des minéraux des sols d'Arcos associées aux pertes du bassin versant fournirait entre 1,51 et $0,75 \mathrm{kmole} \mathrm{ha}^{-1} \mathrm{an}^{-1} \mathrm{de} \mathrm{Si}$, pour un drainage de Si mesuré de $0,89 \mathrm{kmole} \mathrm{ha}^{-1} \mathrm{an}^{-1}$. Cette dernière valeur s'accorderait donc avec une néogénèse mixte de ces deux argiles.

Le rapport $\mathrm{Ca} / \mathrm{Na}$ des échantillons de sols varie autour de $0,2 \mathrm{~g} \mathrm{~g}^{-1}$ à Arcos. Si l'on considère que l'ensemble du
Na libéré par altération est exporté par le ruisseau et dans la biomasse, il vient que l'altération des plagioclases libérerait près de $1 \mathrm{~kg}$ de $\mathrm{Ca}$ à Arcos. Cette valeur est là encore très inférieure au déficit du bilan.

Il semble donc que pour l'ensemble des bassins étudiés, l'altération minérale soit la source de l'essentiel des pertes de cations alcalins. En ce qui concerne le Ca, l'altération des plagioclases ne paraît pas en mesure de compenser ces pertes mais l'altération de l'apatite pourrait jouer ce rôle. L'estimation du flux de Ca libéré par la dissolution de l'apatite apparaît donc indispensable pour juger de la pérennité de ces écosystèmes, comme dans d'autres écosystèmes forestiers sur granite [18].

L'apport d'azote nitrique par les pluies est très faible, entre 0,5 et $1,1 \mathrm{~kg} \mathrm{~N}^{-1} \mathrm{an}^{-1}$. L'azote ammoniacal n'a pas été analysé dans les précipitations. En raison de la proximité de la mer, et de la situation des sites à l'écart de toute forme d'élevage intensif, des dépôts très faibles, égaux à ceux d'azote nitrique peuvent être estimés. Le bilan entrées par les pluies) moins sorties (par drainage et dans la biomasse exportée) est de -6 à $-8 \mathrm{~kg} \mathrm{~N} \mathrm{ha}^{-1}$ $\mathrm{an}^{-1}$. À Jabali et Castrove et $-12 \mathrm{~kg} \mathrm{ha}^{-1} \mathrm{an}^{-1}$ à Arcos. Même si l'on considère des sorties nulles d'azote par drainage telles qu'elles ont été mesurées [20] avant les incendies et coupes, ces bilans demeurent fortement négatifs et une forte fixation symbiotique pourrait seule équilibrer ce bilan.

Les peuplements d'eucalyptus des bassins de Castrove et de Jabali ont été plantés sur d'anciennes landes à ajonc. Cependant l'ajonc disparaît quasi totalement sous les couverts denses des plantations et ne se développe à nouveau que dans les trouées, et à l'occasion des coupes. Par ailleurs les incendies successifs ont certainement été la cause d'importantes pertes d'azote gazeux. À Arcos, l'ajonc ne représente une biomasse notable que sur les crêtes, là ou le peuplement est le moins dense, et dans la trouée ouverte par la coupe. Par mesure de l'activité nitrogénasique de différents sols de lande à ajonc de Bretagne, Rozé $[39,40]$ évalue la fixation symbiotique maximale d'azote en lande haute à $5 \mathrm{~kg} \mathrm{ha}^{-1} \mathrm{an}^{-1}$. Cette valeur correspond à un couvert dense d'ajonc, d'une physionomie proche de celle des landes galiciennes traditionnelles mais très éloignée des sous bois clairs rencontrés. Il est clair que des confirmations locales de cette donnée sont nécessaires, mais on peut légitimement s'interroger sur la pérennité de l'alimentation azotée de ces peuplements.

\section{CONCLUSION}

La présente étude montre que l'exportation de nutriments suivant un mode d'exploitation traditionnel de la 
pinède est supérieure à celle causée par l'exploitation actuelle de l'eucalyptus. Le pin comme l'eucalyptus apparaissent capables d'exploiter de manière très complète les réserves disponibles et les flux fournis par l'altération des minéraux primaires ainsi que les apports par les pluies. Ces derniers jouent d'ailleurs un rôle capital. Parmi les éléments étudiés, ceux potentiellement limitants sont le calcium et l'azote. Dans les bassins versants d'eucalyptus sur granite, le taux extrêmement bas de $\mathrm{Ca}$ échangeable des sols implique une faible libération de $\mathrm{Ca}$ par altération et suggère que la croissance de l'eucalyptus a exploité cette réserve à son profit. Nous avons par ailleurs montré que le prélèvement de $\mathrm{Ca}$ par les racines à partir des horizons profonds ne constituait pas une source importante de Ca pour l'arbre [12]. Il est donc peu probable que l'altération puisse compenser sur la révolution forestière les pertes par exploitation, et ceci d'autant moins que les incendies accélèrent les pertes à la fois par drainage et par transport aérien des cendres. La pérennité de l'alimentation en Ca des peuplements serait mieux assurée si un écorçage en forêt était pratiqué.

Le peuplement de Pin sur granodiorite paraît mieux garanti quant à son alimentation en $\mathrm{Ca}$. Mais des mesures complémentaires sont nécessaires pour préciser la valeur du flux de Ca libéré par l'altération.

Mais c'est l'alimentation en azote des peuplements qui risque d'handicaper à moyen terme leur productivité. Ces forêts sont en effet vraisemblablement en train d'exploiter un stock d'azote précédemment accumulé sous la lande à ajonc. Ce stock n'est pas infini et ceci d'autant plus qu'une partie de l'azote organique est stockée dans une matière organique ancienne, très stable et peu susceptible de se minéraliser $[10,19]$. L'introduction systématique d'essences fixatrices d'azote permettrait sans doute d'éviter de futures carences tout en améliorant la diversité biologique des peuplements.

Les eaux produites par ces bassins versants sont chimiquement appauvries par rapport aux pluies, possèdent une très faible alcalinité et seraient donc a priori très sensibles à une acidification par les dépôts atmosphériques acides. Les bilans du soufre indiquent que ces bassins versants sont à divers degrés capables de retenir les composés soufrés, soit dans la biomasse ligneuse, soit dans les sols sous forme adsorbé ou plus probablement sous forme organique. Dans l'hypothèse d'une augmentation de la pollution atmosphérique acide sur ces sites, des études complémentaires devraient être menées pour analyser les risques.

Remerciements : Ce travail a été mené à l'occasion de l'année sabbatique du premier auteur au Département d'Édaphologie de l'Université de Biologie de Saint Jacques de Compostelle et au Centre de Recherches Forestières de Lourizan, grâce au soutien chaleureux des étudiants, techniciens et chercheurs de ces institutions et à un financement du service des relations internationales de l'INRA.

\section{RÉFÉRENCES}

[1] AFES, Référentiel pédologique, INRA Paris, 1995.

[2] Alvarez E., Martinez A., Calvo R., Geochemical aspects of aluminium in forest soils in Galicia (NW Spain), Biogeochemistry 16 (1992) 167-180.

[3] Aulenbach B.T., Hooper R.P., Bricker O.P., Trends in the chemistry of precipitation and surface water in a national network of small watersheds, Hydrological processes 10 (1996) 151-181.

[4] Bara S., Estudio sobre Eucalyptus globulus. I Composition mineral de las hojas en relacion con su posicion en el arbol, la composicion del suelo y la edad, IFIE comunicacion, 67 (1970).

[5] Bara S., Rigueiro Rodriguez A., Del Carmen Gil Sotres M., Mansilla Vasquez P., Alonso Santos M., Efectos ecologicos del Eucalyptus globulus en Galicia, INIA, Madrid, 1985.

[6] Bellido F., Brandle J.L., Lasala M., Reyes J., Consideraciones petrologicas y cronologicas sobre las rocas graniticas hercinicas de Galicia, Cuaderno Laboratorio Xeoloxico Laxe 17 (1992) 241-261.

[7] Bouhier A., La Galice. Essai géographique d'analyse et d'interprétation d'un vieux complexe agraire, Imprimerie Yonnaise, La Roche sur Yon, 1979.

[8] Buldgen P., Étude écosystémique de deux bassins versants boisés de Haute Ardennes, Thèse, Université Liège, 1984.

[9] Calvo de Anta R., El eucalipto en Galicia, Santiago de Compostela University Press, 1992.

[10] Carballas M., Carballas T., Jacquin F., Biodegradation and humification of organic matter in humiferous atlantic soils, Anales Edafologia Agrobiologia 38 (1979) 1699-1717.

[11] Courcoux P., Approche du cycle biologique d'un écosystème à pin maritime en lande humide, DEA INRAUniversité Bordeaux, 1982.

[12] Dambrine E., Loubet M., Vega A., Lissarague A., Localization of mineral uptake by deep tree roots using $\mathrm{Sr}$ isotopes, Plant. Soil. 192 (1997) 129-132.

[13] Décourt N., Tables de production pour les forêts françaises, ENGREF Nancy, 1973.

[14] Durand P., Biogéochimie comparée de trois écosystèmes (pelouse, hêtraie, pessière) de moyenne montagne granitique (Mt Lozère). Thèse, Université Orléans, 1989.

[15] Durand P., Lelong F., Neal C., Comparison and significance of annual hydrochemical budgets in three small granitic catchments with contrasting vegetation (Mt Lozère) Environ. Pollut. 75 (1992) 223-228.

[16] FAO, Soil map of the world. Revised legend, FAO, Rome, 1990.

[17] Fernandez Lopez A., Modelos de prediccion de la evolucion de la productividad en los turnos sucesivos de eucalipto 
tratado a monte bajo. Publicacion Interna Centro de Investigacion Forestal de Lourizan, 1994.

[18] Fichter J., Dambrine E., Turpault M.P., Ranger J., Base cation supply in spruce and beech ecosystems of the Strengbach catchments (Vosges Mountains, NE France). Water, Air, Soil Pollut. 104 (1997) 125-148.

[19] Gonzales-Prieto S.J., Carballas T., Composition of organic N in temperate humid regions soils (NW Spain), Soil Biology and Biogeochemistry 9 (1991) 887-895.

[20] Gras J.M., Investigacion sobre las plantaciones de Eucalyptus globulus en Galicia, Thesis, Universidad Madrid, 1993.

[21] Gras J.M., Vega J.A., Bara S., Six years of study on fast growing forests plantations in the Northwest of Spain, Acta Geologica Hispanica 11 (1995) 25-36.

[22] Grip H., Bishop K., Chemical dynamics of an acid stream rich in dissolved organics. in: Mason B.J. (Eds.), The Surface Water Acidification Programme, 1990, pp. 75-83.

[23] Grip H., Malmer A., Wong F.K., Converting tropical rainforest to forest plantations in Sabah, Malaysia. Part I. Dynamics and net losses of nutrients in control catchments streams, Hydrological Proc. 8 (1994) 179-194.

[24] Guitian Ojea F., Carballas T., Suelos de la zona humeda espanola, III Ranker atlantico, Anales de Edafologia y Agrobiologia 27 (1968) 57-73.

[25] Hedin L.O., Armesto J.J., Johnson A.H., Patterns of nutrients loss from unpolluted, old growth temperate forests: evaluation of biogeochemical theory, Ecology 76 (1995) 493509.

[26] Hornung M., Roda F., Langan S.J., A review of small catchment studies in Western Europe producing hydrochemical budgets, Air Pollution Research Report 28. EEC, Brussels, 1990.

[27] Joder A., Las clacificaciones en Edafologia, Anales Edafologia y Agrobiologia 26 (1969) 12-44.

[28] Jordan C.F., The nutrient balance of an Amazon rain forest, Ecology 63 (1982) 647-654.

[29] Keller R., Caractéristiques du bois de pin maritime. Variabilité et transmission héréditaire, Ann. Sci. For. 30 (1973) 31-62.

[30] Lemoine B. Ranger J., Gelpe J., Distribution qualitative et quantitative des éléments nutritifs dans un jeune peuplement de Pin maritime (Pinus pinaster Ait), Ann. Sci. For. 45 (1988) 95-116.

[31] Lemoine B., Gelpe J., Ranger J., Nys C., Biomasse et croissance du pin maritime. Étude de la variabilité dans un peuplement de 16 ans, Ann. Sci. For. 43 (1986) 67-84.

[32] Lesack L.F.W., Melack J.M., Mass balance of major solutes in a rainforest catchment in the central Amazon: implications for nutrient budgets in tropical rainforests, Biogeochemistry 32 (1996) 115-142.
[33] Macias F., Chesworth W., Weathering in humid regions, with emphasis on igneous rocks and their metamorphic equivalents, in: Martini L. \& Chesworth W. (Eds.), Weathering, Soils and Paleosols, Elsevier, Amsterdam, 1992, pp. 283-306.

[34] Macias F., Guitian Riviera F., Garcia-Rodeja E., Vidal Romani J.R., Componentes no cristalinos y cristalinos (gibbsita y caolinita) en los productos de neoformacion de rocas graniticas de Galicia, Cuaderno Laboratorio Xeoloxia Laxe 1 (1980) 51-67.

[35] Madeira M., Influence of mineral nutrient redistribution in Eucalyptus plantations on soil properties in: Berthelin J. (Eds.), Diversity of Environment Biogeochemistry, Elsevier, Amsterdam, 1991, pp. 485-494.

[36] Probst A., Lelong F., Viville D., Durand P., Ambroise B., Fritz B, Comparative hydrochemical behaviour and element budget of the Aubure and Mt Lozere catchments. in: Landmann G., Bonneau M. (Eds.), Forest Decline and Atmospheric Deposition Effects in the French Mountains, Springer Verlag, Berlin, 1995, pp. 203-222.

[37] Robert M., Principes de détermination qualitative des minéraux argileux à l'aide des rayons X. Ann. Agronom. 26 (1975) 363-399.

[38] Rosen K., Supply, loss and distribution of nutrients in three forest watersheds in central Sweden, Thesis, Uppsala University, 1982.

[39] Rozé F., Le cycle de l'azote dans les landes bretonnes, Thèse Université Rennes, 1986.

[40] Rozé, F., Variations de la fixation d'azote atmosphérique liée à Ulex europaeus dans les landes de Bretagne, Rev. Ecol. Biol. Sols 25 (1988) 279-286.

[41] Soalleiro R.R., Alvarez J.G., Vega G., Modelo dynamico de crecemento de masas regulares de Pinus pinaster Aiton en Galicia. Xunta de Galicia, Santiago, 1994.

[42] Taboada T., Romero R., Garcia C., Weathering evolution of a biotite granite (El Pindo, Galicia, NW Spain), Chemical Geology 84 (1990) 130-132.

[43] Tardy Y., Géochimie des altérations. Étude des arènes et des eaux de quelques massifs cristallins d'Europe et d'Afrique, Thèse Université L. Pasteur, Strasbourg, 1969.

[44] Tomé M., Ribeiro F., Soares P., Pereira H., Miranda I., Pina J.P., Effects of spacing on eucayptus globulus fibre yield and quality. in: Eucalypt plantations: Improving Fibre Yield and Quality, C. R Coll. IUFRO, Hobard, 1995, pp. 60-62.

[45] Ugolini F.C., Corti G., Agnelli A., Piccardi F., Mineralogical, physical, and chemical properties of rock fragments in soil, Soil Sci. 161 (1996) 521-542.

[46] Wright R.F., Norton S.A., Brakke D.F., Frogner T., Experimental verification of episodic acidification of freshwaters by sea salts, Nature 334 (1988) 422-424. 